\title{
LOS CONSEJOS VERBALES DE GUERRA FRENTE A LA TEORÍA DE LA JUSTICIA. UN ESTUDIO DE CASO *
}

\section{Verbal court-martial regarding the theory of justice. A case}

\author{
Henry Torres Vásquez** \\ Eder Maylor Caicedo Fraide ${ }^{* * *}$ \\ Daniel Alfonso Barragán Ronderos**** \\ Universidad La Gran Colombia. Bogotá, D.C.
}

"Quien investiga, debe ser el mismo que falla»; "es preferible condenar a un inocente que absolver a un culpable (...) para evitar las dilaciones de la justicia

ordinaria, habría que convertir el indicio en prueba suficiente para condenar a un sindicado ${ }^{1}$ (Alternativa. Nro 67. Enero 26 a febrero 2 de 1976, p. 3.)

Recepción: 23 de marzo de 2015. Aceptación: 19 de junio de 2016.

DOI: http://dx.doi.org/10.21017/Rev.Repub.2016.v21.a12

* Artículo que hace parte del trabajo integrado entre los grupos de investigación Familia, Conflictos Sociales y Proyección Social, Teoría Política y del Derecho y Derecho Penal y Sistema penal acusatorio de la Universidad La Gran Colombia, sede Bogotá. A su vez, el artículo se encuentra inscrito en el proyecto El terrorismo en el Sistema Interamericano de Derechos Humanos.

** Doctor en Sistema penal de la Universidad Jaime I de Castellón, España. Tesis doctoral: Análisis del terrorismo de Estado, máxima calificación cum laude por unanimidad, 2008. Abogado de la Universidad Nacional de Colombia. Par académico e investigador asociado (1) de Colciencias. Docente investigador de tiempo completo de la Facultad de Derecho de la Universidad La Gran Colombia, Bogotá, D. C. Líder del grupo de investigación Derecho Penal y Sistema penal acusatorio, categoría C de Colciencias. Correo electrónico: henry.torres@ugc.edu.co

*** PhD en Historia de la Universidad Nacional de Colombia. Magíster en Sociología y politólogo de la misma institución. Actualmente, doctor-investigador de la facultad de Derecho de la Universidad La Gran Colombia. Consultor de la Unidad de Restitución de Tierras-Ministerio de Agricultura. Líder del grupo de investigación: Familia, conflictos sociales y proyección social. Reconocido por Colciencias, categoría C. Correo electrónico: eder.caicedo@ugc.edu.co

**** Coordinador de Investigaciones de la Facultad de Derecho de la Universidad La Gran Colombia. Miembro del grupo de investigación Teoría del Derecho, la justicia y la política, reconocido por Colciencias, categoría D. Proyecto de investigación: La construcción democrática inmersa en la Constitución de 1991. Correo electrónico: daniel.barragan@ ugc.edu.co

1 Fiscal, mayor Gerardo Ñungo. Referido al caso de los sindicados del asesinato del general Ramón Arturo Rincón Quiñones. 


\section{RESUMEN}

El presente artículo estudia los Consejos Verbales de Guerra a través de la Teoría de la justicia de John Rawls, considerando el desarrollo de los principales acontecimientos en un seguimiento exhaustivo de la prensa y la opinión periodística de la revista Alternativa y el diario El Tiempo, para el periodo 1974-1978.

Consolida el pensamiento de que la justicia está estrechamente relacionada con la democracia, caracterizando a los estados de sitio como espacios antidemocráticos por la falta de consenso social y político, en la implementación de los mismos, por parte de los gobiernos de la época del Frente Nacional, y posteriormente, en la presidencia de Alfonso López Michelsen.

Analiza la forma como se ha aplicado la política de seguridad nacional en la región latinoamericana, especialmente en Colombia, llegando a una serie de entendimientos sobre la forma en que EE. UU. ha venido interviniendo en la región y ha ocasionado la debacle por las acciones militares financiadas en varios gobiernos que tienen una naturaleza antidemocrática.

Se reflexiona sobre la forma en que se define el delito político en Colombia, considerando al estado de sitio y a los consejos verbales de guerra, como espacios proclives a inculpar ciudadanos, sin los debidos parámetros de justicia, entrando en la violación sistemática de los derechos humanos.

Finalmente, se presentan unas conclusiones con relación a los resultados emanados del estudio de caso y del filtro que constituye la Teoría de la justicia de John Rawls, además de su cuerpo de obras de contenido filosófico político, advirtiendo una serie de desarrollos prospectivos para alcanzar la democracia, necesaria para llegar a la verdadera justicia.

Palabras clave: Consejos Verbales de Guerra, teoría de la justicia, estado de sitio, Colombia.

\section{ABSTRACT}

This article studies Verbal war councils through the Theory of Justice of John Rawls, considering the development of major facts in a comprehensive monitoring of the press and journalistic opinion of the Alternativa magazine and the newspaper El Tiempo, for the period between 1974 to 1978.

It consolidates the reasoning that justice is closely related to democracy, characterizing the state of siege as anti-democratic space for the lack of social and political consensus in the implementation thereof, by the governments at 
that time of Frente Nacional and onwards, Alfonso López Michelsen presidency.

It also analyzes how national security policy has been applied in Latin America, especially in Colombia, reaching a series of understandings on how U.S.A. has been intervening in the region and caused the debacle by military actions, financed in several governments that have an anti-democratic nature.

The way that political offences is defined in Colombia is also took it into consideration. This to consider the state of siege and Verbal war councils as scenarios prone to indict citizens, without proper standards of fairness, fostering a systematic violation of human rights spaces.

Finally, conclusions are presented in relation to the issued results of the case study and filter which is the theory of justice of John Rawls, in addition to his mainstream of political philosophical content, to indicate a number of prospective developments for achieving true democracy, needed to reach true justice.

Keywords: Verbal court-martial, theory of justice, state of siege, Colombia.

\section{INTRODUCCIÓN}

La esperanza de cambio que mantenían los sectores populares con la administración de Alfonso López Michelsen (1974-1978), rápidamente fue defraudada con medidas económicas antipopulares que dejaron ver las intenciones de continuidad con el modelo del Frente Nacional. Las alzas en los productos básicos de la canasta familiar se convirtieron en una constante, que cada año, generaba protestas y reclamos por parte de los sectores subalternos -situación similar a la vivida durante los años inmediatamente anteriores-. Justamente, el año siguiente de la posesión de López fue de los más movidos en materia de protestas sociales y huelgas obreras del periodo estudiado.

Durante el primer semestre de 1975 se presentaron de manera escalonada paros en los sectores bancario y cementero; asimismo a mitad del año se desarrollaron huelgas en el magisterio, a las que les siguieron paros en el sector de la salud. A esta serie de huelgas se sumaron: «la huelga de trabajadores de Prefabricados S. A., la cual completó a final de 1975 siete meses contando con la presencia de 75 trabajadores y la huelga de Calzado Bronx que a final de año completaba 6 meses de toma por parte de los 139 trabajadores y que los dueños habían cerrado a finales de año argumentando la quiebra de la empresa» (Alternativa. Nro. 65. Diciembre 26 de 1975-enero 6 de 1976, p. 22). 
Una de las más notorias fue la desatada en el ingenio de Riopaila, en el Valle del Cauca, que empezó paulatinamente en marzo de 1975 y desencadenó en un paro de grandes magnitudes, prolongado hasta marzo del año siguiente; esta huelga contó con una nutrida presencia de trabajadores y fue una de las más reprimidas por parte del establecimiento. La huelga de trabajadores del ingenio de Riopaila dejó dos muertos y varios trabajadores despedidos. Sin embargo, la represión también se observó en los medios de comunicación, a los cuales se les prohibió informar sobre los acontecimientos ocurridos en Riopaila.

A finales de diciembre la revista Alternativa informaba sobre la renuncia de 6 redactores del periódico El Pueblo de Cali, en protesta por la actitud asumida por el director del diario, Mario Rengifo -catalogado como Liberal-Llerista-, quien se negó a dejar publicar información en el periódico sobre el desarrollo de la huelga de Riopaila. De igual forma, denunció Alternativa que «ni ese diario, ni los otros del Valle, «El Occidente» de los Caicedo y «El País» de los Lloreda, ni ningún otro diario «serio y responsable» han vuelto a informar sobre un conflicto social de tan grandes proporciones». (Alternativa. Nro. 65. Diciembre 26 de 1975-enero 6 de 1976, p. 22).

La medida de choque implementada por el gobierno de forma inmediata fue la imposición por decreto del estado de sitio, el cual instauró al año de su posesión. La benevolencia con el movimiento sindical y popular con la cual había iniciado el gobierno, rápidamente se transformó en medidas represivas conducentes a limitar la protesta social.

En efecto, con el inicio del gobierno de López Michelsen la represión había adquirido un carácter preventivo y selectivo, es decir, dirigida principalmente a los líderes de las organizaciones sociales; empero, el ascenso del descontento popular, incontenible en su magnitud, llevó al gobierno a una cada vez mayor escalada represiva, que empezaría a tener un carácter masivo y generalizado.

Uno de los análisis más interesantes en cuanto a las medidas represivas tomadas al año de posesión de López es el «Informe del Comité de Solidaridad con los Presos Políticos ante el Tribunal Russell», en el que se hace énfasis en dos decretos presidenciales, posteriores a la instauración del Estado de Sitio, el 26 de junio de 1975. El primero es el decreto 1533 de 1975, implementado el 6 de agosto y que según el informe

Busca impedir cualquier forma de protesta popular, así sea la más elemental como la expresión moral. Aumenta las sanciones para los dirigentes e impone multas en caso de huelgas; incita a la conformación de grupos de civiles para que señalen y detengan a los infractores de esta norma; decreta los juicios colectivos y se limita a 10 minutos el derecho universal de defensa; se atenta contra 
el derecho de huelga; contra la libertad de conciencia y opinión [continúa el informe afirmando que entre agosto de 1975 y enero de 1976]... se han montado (sic.) más de 28 juicios colectivos con cerca de 500 condenados a sanciones que fluctúan entre 3 días y 6 meses de cárcel (Alternativa. Nro. 65. Diciembre 26 de 1975-enero 6 de 1976, p. 1).

El otro decreto al que se hace referencia es el 2407, promulgado en el mes de noviembre del mismo año, el cual «elimina en la práctica el delito de rebelión que es político... dándole el carácter de asociación para delinquir a todo «acto preparatorio» que atente contra el orden público» (Alternativa. No. 65). Este último decreto cobija desde un paro cívico, hasta la preparación de una huelga y se destaca en el informe que «en la práctica los presos políticos vienen siendo condenados por asociación para delinquir, que tiene penas hasta de 14 años, o sea que se trata de la mera formalización de una arbitrariedad jurídica que la justicia militar de todos modos comete» (Alternativa. Nro. 65). En cuanto a lo que tiene que ver con el delito de Rebelión, es menester entender que para que se configure dicho delito, «se debe presentar la toma de las armas para subvertir el orden establecido con el fin de obtener una mejora en las condiciones de vida de los habitantes de una nación, en otras palabras dicho delito persigue la reivindicación política real o presunta» (Chaparro, 2013, p. 19).

De otra parte, muchos de los regímenes dictatoriales de Latinoamérica se han caracterizado por la violación a los derechos humanos que se lleva a cabo de forma generalizada y sistemática, fundamentada en políticas de Estado; de ahí que luego de haber pasado el transcurso de la dictadura muchas de esas graves violaciones salen a la luz pública. Es igualmente conocido que para evitar juzgar a los responsables, que mayoritariamente son agentes estatales, se crean leyes con el fin de que los delitos queden impunes, lo cual es considerado por Torres un terrorismo de Estado (2010).

Así, pues, uno de los grandes mecanismos de impunidad han sido las llamadas leyes de autoamnistía, destinadas a que no haya sanción alguna para los responsables. Tal es el caso de Almonacid Arellano, el caso Barrios altos, caso Gelman vs. Uruguay, actos que resultan incompatibles con la Convención Americana de Derechos Humanos, señalando además que esta al adherirse a la convención se presume un compromiso de los Estados partes para respetar los derechos humanos, dar fuertes garantías de procesos judiciales y ejercer el libre ejercicio de los derechos de la sociedad.

El resto del continente no fue ajeno a estas prácticas. Casos como el de Almonacid Arellano y otros contra Chile, debatido en la Comisión Interamericana de Derechos Humanos (CIDH), ponen de manifiesto que en Chile, a través del Decreto Ley nro. 2.191 de 18 de abril de 1978, se permitía la 
impunidad para los responsables de abominables crímenes de lesa humanidad. Esto era posible en la medida en que se concedía amnistía a todas las personas que en calidad de autores, encubridores o cómplices hubiesen cometido actos delictuosos con ocasión del estado de sitio vigente entre el 11 de septiembre de 1973 y el 10 de marzo de 1978, con la única condición de que no se encontraran sometidas a un proceso o condenadas por otros actos.

El Tribunal, como conclusión de todo lo señalado, «considera que el asesinato del señor Almonacid Arellano formó parte de una política de Estado de represión a sectores de la sociedad civil, y representa solo un ejemplo del gran conjunto de conductas ilícitas similares que se produjeron durante esa época, este aspecto demuestra cuán extendida estaba la doctrina de la seguridad nacional en el continente» (CIDH, Sentencia 26 de septiembre 2006, argumento nro. 151).

Retornando el caso colombiano, en procura de analizar teóricamente la situación vivida en el periodo presidencial de 1974-1978 se cita la obra de Rawls $(1971)^{2}$ que depara una comprensión de ilegitimidad del poder político que genera un conflicto social, base de la desolación injusta que penetra la vida integra de la nación andina.

En este estado de cosas, la teoría de la justicia de John Rawls (1971) sirve para ser aplicada en el ámbito nacional como doctrina jurídica, que se consolide en la defensa de los derechos humanos, en busca de la igualdad, la libertad y el respeto a la vida, a favor de un estudio que descubra la falta de legitimidad del Estado colombiano.

Para tal efecto, es necesario revisar los conceptos teóricos de Ralws, su aplicación en el caso histórico anteriormente desarrollado y plantear soluciones desde dicha teoría a problemáticas cotidianas de la realidad colombiana.

John Rawls defiende una visión más social y moral de la política, considerando los principios que desarrolla:

El primer principio es el de igual libertad, que trata del «debido funcionamiento de las instituciones que permita que toda persona tenga un derecho igual al conjunto más amplio de libertades que sea compatible con un conjunto seme-

2 En su obra, Rawls expresa el sentido de la justicia, que se inspira en la compresión social que se le da a un ordenamiento, que en cuanto a características éticas, morales y en torno a la virtud, aduce a un proceder coherente con la forma de vida que se ha desarrollado en la sociedad, es decir, la responsabilidad que es atinente a la autoridad al provocar el mejor de los ordenamientos político-jurídicos. 
jante de libertades para todos», el segundo principio está comprendido en dos partes: a) El de igualdad equitativa de oportunidades, que se refiere a que las «oportunidades deben estar vinculadas a funciones y posiciones a las cuales todos tengan igual acceso, dados los talentos requeridos», y b) el de la diferencia, que trata sobre «la creación de condiciones que deben servir para el mayor beneficio de los menos aventajados de la sociedad, de acuerdo a lo generado en la posición original» (Botero, 2004).

De estos principios rawlsianos se desprende el sentido de la justicia y, por ende, la compresión de la legitimidad del poder del Estado, porque desde los planteamientos de equidad que expresa la teoría de la justicia, los principios de la justicia y la concepción de los bienes básicos, se expresa la construcción social y consensual del Estado.

A esto se suma, que «Rawls dedique gran parte de las primeras páginas de su Teoría de la justicia a la crítica del utilitarismo y el intuicionismo, dejando claro que se propone ofrecer una alternativa a ambos» (Contreras, 2009, 138), lo cual trasciende en el orden de los gobiernos porque se superan políticas de mayor eficacia (a nivel de utilidad del gobierno de turno) por políticas democráticas que en consenso determinen formas justas de gobernar. La alternativa consiste en volver a colocar al contractualismo en las primeras instancias del pensamiento político, estableciendo nuevos parámetros para su comprensión, en pro de la justicia social. La idea fundamental es proteger al pueblo soberano autogobernado a través del establecimiento legal de una constitución que, como carta de principios y derechos humanos, debe ser respetada en todo momento y por todo gobierno, haciendo inconcebible estados de sitio que fragmenten el consenso establecido como contrato social en la carta política. Cuestión olvidada en el levantamiento de los juicios verbales de guerra en contra de la sociedad, sin un enemigo externo, acusando al mismo soberano en sus integrantes y degradando el orden de lo justo, vulnerando todos los derechos humanos. Solo por indicios mal concebidos, acusaciones infundadas, malas administraciones y políticas perversas se llega a la ignominia de acusar al inocente, sentenciar sin derecho a queja, y a violar todos los parámetros de lo que se entiende como una verdadera democracia.

Cabe decir que la demagogia como forma de gobierno es la visión corrupta de la democracia ${ }^{3}$. La demagogia es mentir de manera descarada para conseguir un fin individual, estableciendo en la mentira un fin social. Esto se ve reflejado en las consecuencias que sufrió la sociedad colombiana, en el primer año de gobierno de Alfonso López Michelsen, que traicionó la visión social de su cam-

3 Como la interpretara Aristóteles en su famoso tratado sobre Política (1991). 
paña, por la visión corrupta de un gobierno apoderado de los recursos, que impone el ordenamiento político y jurídico en contra de la sociedad, que de por sí, fue la forma de gobernar al país en el periodo del Frente Nacional, en el periodo posterior, y aún hoy en día, cuando candidatos presidenciales suben al poder con promesas realizadas en campaña que incumplen al momento de ingresar al gobierno por seguir políticas internacionales que solo benefician a las potencias internacionales, principalmente al país del norte.

\section{PLANTEAMIENTO DEL PROBLEMA DE INVESTIGACIÓN}

En Colombia, los Consejos Verbales de Guerra fueron habituales en cuanto al juzgamiento de civiles acusados de delitos que atentaban presuntamente contra el orden constitucional y legal del régimen democrático o la seguridad pública. Desde agosto de 1949 hasta 1991 Colombia vivió permanente bajo la figura del estado de sitio, generada por la aplicación del artículo 121 de la Constitución (de 1886) en la que por motivos de conmoción interior, podía el presidente, previa audiencia del Consejo de Estado y con la firma de todos los ministros, declarar turbado el orden público y en estado de sitio toda la República o parte de ella.

Esta situación debilitó las instituciones, ya que el ejecutivo legislaba y tenía facultades desmedidas que rayaban en el autoritarismo. Es, pues, «la provocación de un claro desbalance constitucional en el que la fuerza pública excedía sus poderes» (Tirado, 1978, 164), el escenario en el que la sociedad civil postulaba sus reivindicaciones. Buena parte de ese periodo, que comprende el Frente Nacional y el pleno auge de las guerrillas, incidió en la legitimación de las arbitrariedades de los militares. Esta situación era peor cuando se trataba de combatir las acciones de los grupos guerrilleros en virtud de la competencia asignada a los militares respecto a poder llevar procesos penales en los que estuvieren involucrados civiles en ciertos delitos que afectaban las instituciones estatales. En ese sentido, los delitos políticos tienden a desaparecer, dando paso a que las conductas ejecutadas por detractores del Estado fueran catalogadas como delitos atroces, realizados con ferocidad y barbarie según la consideración de los jueces que integraban los Consejos Verbales de Guerra.

En los momentos de profunda crisis en Colombia -y el periodo del «Frente Nacional» no fue la excepción- aparece la necesidad de buscar una verdadera democracia que se ha ausentado de la vida política de esta sociedad. Bajo el prisma teórico, la situación presentada en el Frente Nacional se estudia con la Teoría de la Justicia de John Rawls (1971), en la cual se presenta un análisis de las condiciones que brinda la Justicia para la estabilidad socio-política. 
Entendiendo que en el periodo del Frente Nacional se generó una inusitada inestabilidad social y política, con un desbalance entre la autoridad y el poder, que a todas luces representa un ordenamiento político-jurídico no legítimo y la falta de aceptación popular de medidas que atentan contra los derechos humanos, como se observa en los Consejos Verbales de Guerra, se evidencia un conflicto entre el Estado representativo con la sociedad civil que lo ha elegido, rompiendo el paradigma del consenso, base de la democracia.

En una democracia, «El sentido de la justicia» (Rawls, 1971, p. 410) aporta la estabilidad de una sociedad bien ordenada. El aporte rawlsiano se configura en la forma como la legitimidad de la autoridad es establecida por el sentido de justicia y la coherencia que este posee en la forma como se ejerce el poder. La verdadera democracia alude a un estado de cosas igualitario, libertario y justo, que cumpla con los principios consolidados por la sociedad en un consenso traslapado. Es decir, en común-unidad la actuación del Estado va a favor de los parámetros establecidos por todos y no en contravía de los mismos, considerando que la democracia no se impone-como en un decreto de Estado de sitio- sino que se construye a diario, llegando a acuerdos que posibiliten la convivencia entre los distintos. La percepción que hace John Rawls (1971) permite configurar una democracia creada desde abajo, por todos en común-unidad y no concibiendo a la democracia como la forma efectiva de un gobierno.

En el Estado colombiano la democracia siempre ha sido cuestionada, especialmente desde consideraciones referidas a la justicia, donde fue evidente, en aquella época, la violación masiva a derechos humanos. Torres (2009) señala que «en Colombia el predominio del militarismo frente a cualquier otra posibilidad no violenta es de facto jerárquicamente igual a cualquier rama del poder público, e incluso, en múltiples ocasiones superior a ellas. (...) Por supuesto, que el principal apoyo financiero a las fuerzas militares son los EE. UU., estos sostuvieron el imperialismo y del mantenimiento del statu quo, sin importar para nada, los medios o los métodos utilizados» (pp. 19-21). Es decir, el accionar de conductas de parte de agentes del Estado permitió la violación a derechos humanos.

En un mundo en el cual los principios básicos son democráticos, se tendrán como mínimos éticos, los derechos humanos fundamentales, para la dignidad y para el sostenimiento del sujeto y la sociedad. Así se verán cumplidos los criterios teóricos de Rawls en su «teoría de la justicia» (1971), su «liberalismo político» (1996) y su «derecho de gentes» (1998).

De aquí podemos extraer la pregunta: ¿Los Consejos Verbales de Guerra en Colombia durante el Frente Nacional hacían parte de la injusticia del Estado? 


\section{METODOLOGÍA}

La naturaleza de este artículo es histórica y teórica, hacia el estudio de caso, en la prospectiva de generar nuevos entendimientos con respecto a la relación entre democracia y justicia.

Esto se consolida, aplicando un enfoque socio-crítico en el que se combinan aspectos históricos, jurídicos y sociales, utilizando el método de análisis-síntesis unido al lógico-teórico, y lógico-racional, el que básicamente se guía por una estrategia deductiva e inductiva. Con lo cual, se allega una serie de información que una vez sistematizada permite obtener unos resultados que dan lugar a un estudio de caso. Como lo concibe la profesora Raquel Martínez (2007):

La línea de investigación socio-crítica puede llegar a combinar los planteamientos de las líneas de investigación empirista-positivista, de carácter cuantitativo, y etnográfica, de carácter cualitativo, que representan posiciones metodológicas muy diferenciadas. (...) Por ello, esta línea de investigación incorpora, complementándolos, los objetos de estudio de la línea empirista-positivista y de la línea etnográfica. (...) Persigue, además, conocer las «teorías implícitas» con las que las personas interpretan, predicen y actúan en el mundo social para crear un conocimiento colectivo que sea expresado en un lenguaje cotidiano para que pueda ser fácilmente comprendido y también útil al mayor número posible de personas (p. 33).

En definitiva, la metodología empleada en el estudio de caso pasa por la comprensión de los postulados de la Teoría de la justicia de John Rawls, que argumenta desde el contractualismo la forma como se comprende la democracia a partir del consenso traslapado. Este prisma de análisis teórico presenta una ventaja para determinar el sentido de justicia y entender cómo los estados de sitio se consolidan como espacios antidemocráticos de gobierno, que fueron un común denominador en el periodo de 1949 a 1991, cuando el mundo vivía lo que los historiadores han considerado como la Guerra Fría.

\section{EL ESTADO DE SITIO Y EL AMPARO DE LA SEGURIDAD NA- CIONAL DURANTE EL «FRENTE NACIONAL» EN COLOMBIA}

Los estados de sitio como mecanismo que otorga facultades extraordinarias al gobierno han sido en la historia de Colombia un instrumento importante para fomentar la represión ciudadana, fueron muy bien manejados por los diferentes gobiernos en lo que fue todo el siglo pasado, del mismo modo florecieron 
en la etapa del Frente Nacional, «utilizados principalmente en las ciudades contra los movimientos sociales que estaban desestabilizando un régimen político frágil que luchaba para superar la crisis económica y política» (Iturralde, 2003, p. 33). Es de singular importancia para la línea argumentativa de este trabajo determinar que uno de los principales «beneficiados» es la fuerza pública, a quien se le dota de instrumentos penales sustantivos, pero sobre todo procesales, para solventar, entre otros, problemas suscitados por la sociedad en materia de seguridad.

Por su parte el estado de sitio se hallaba en Colombia en la Constitución Nacional (1886); en el artículo 121 se indicaba que:

En los casos de guerra exterior, o de conmoción interior, podrá el Presidente, previa audiencia del Consejo de Estado y con la firma de todos los Ministros, declarar turbado el orden público y en estado de sitio toda la República o parte de ella.

Mediante tal declaración quedará el Presidente investido de las facultades que le confieran las leyes, $y$, en su defecto, de las que le da el Derecho de gentes, para defender los derechos de la Nación o reprimir el alzamiento. Las medidas extraordinarias o decretos de carácter provisional legislativo que, dentro de dichos límites, dicte el Presidente, serán obligatorios siempre que lleven la firma de todos los Ministros.

El Gobierno declarará restablecido el orden público luego que haya cesado la perturbación o el peligro exterior; y pasará al Congreso una exposición motivada de sus providencias. Serán responsables cualesquiera autoridades por los abusos que hubieren cometido en el ejercicio de facultades extraordinarias.

Ahora bien, para entender la dinámica del Frente Nacional es necesario recordar el contexto latinoamericano en materia de los militares en el poder o detrás del mismo. En ese momento, como señala Torres (2009), «la seguridad era crucial para la paz continental»; en esa búsqueda, el enfrentamiento constante entre las fuerzas militares, legalmente constituidas por el Estado, y movimientos insurgentes, además de la delincuencia común, terminó propiciando una lucha por el poder.

\section{EL ESTADO DE SITIO: IMPACTOS DE LA POLÍTICA ESTADO- UNIDENSE EN LATINOAMÉRICA}

Como se viene presentando, el autoritarismo latinoamericano era un escenario proclive para el empoderamiento de los militares en la región. La situación era 
tal que en 1968, el 62\% de los Estados en el mundo eran dictaduras militares; por supuesto que gran parte de esa cifra se la llevaba este continente.

Ciertamente, los estados de sitio se amparaban en la Seguridad Nacional, concepto que provino de la doctrina de su mismo nombre en los EE. UU.; allí «se refiere a la defensa nacional (...), a la protección de la República contra todos los peligros internos o externos».

Esta doctrina surgió en América Latina hacia 1960; un autor como De Roux (2000, p. 137) llega a conceptuar que bajo la influencia de la ideología de la Seguridad Nacional se violaron los derechos humanos contra miembros reales o imaginarios de grupos subversivos. Bajo ese imperio de seguridad nacional, la fase de formación se hizo en la «Escuela de las Américas» (Barker, 2004, p. 80). Esta tuvo entre sus alumnos a Omar Torrijos de Panamá, quien tomó parte en el golpe militar que depuso al presidente Arnulfo Arias en 1968; Manuel Antonio Noriega, después de la muerte de Torrijos en un accidente aéreo ocurrido en 1981, se convirtió en jefe del Estado Mayor y obviamente llegó a tener el control efectivo del gobierno panameño; Anastasio Somoza de Nicaragua, quien fuera el patriarca de una dinastía de dictadores, los cuales, con el apoyo de Estados Unidos, gobernaron a Nicaragua durante 43 años; Leopoldo Fortunato Galtieri de Argentina, que formara parte del grupo militar que derrocó a la presidenta Isabelita Martínez de Perón (1974-1976) y fue miembro de la Junta Militar que ejerció la dictadura bajo el gobierno del general Roberto Viola; en diciembre de 1981 fue designado presidente de la República por la Junta Militar.

Debido a que sus alumnos fueron en muchas ocasiones denominados «sanguinarios» (Barker, 2004, p. 80), en el 2001 se le cambió el nombre a la escuela por el de Instituto del Hemisferio de Occidente para la Cooperación en Seguridad (WHISC por sus siglas en inglés).

Llegando a los principios teóricos rawlsianos, para analizar las consecuencias de la política de seguridad nacional que proviene de los EE. UU. y se afirma a nivel internacional sobre Latinoamérica, la teoría de la justicia tiene una expresión global en la obra El derecho de gentes (1998), la cual se inscribe en el plano internacional, considerando, «[E]n primer lugar, el principio de igualdad. Este principio establece que los distintos pueblos, constituidos en Estados, poseen derechos iguales. El primero de ellos, y el más importante, es el de autodeterminación». (Pérez, 2009, 110).

En cuanto a la autodeterminación de los Estados, cuyo propósito es garantizar al pueblo soberano las garantías del orden constitucional, planteado en consenso, la política de seguridad nacional que gobernó a Latinoamérica evitó 
que democráticamente lo expresado en las constituciones se llevara a cabo, y más bien, con el propósito de beneficiar al país del norte, EE. UU. -como aún se sigue haciendo-, los gobiernos se olvidaran de sus pueblos, a quienes debían su poder, y empezaran a forjar un estado de cosas inconstitucionales, vulnerando derechos humanos y arrancando las posibilidades de construcción pacífica de la sociedad civil, sumergiéndonos en un profundo conflicto, que persiste en Colombia, como lo expresa Barragán (2011): « el fuerte posicionamiento militar estadounidense a nivel mundial, en intervenciones marcadas en territorios de países como Arabia Saudita, Palestina, Colombia, entre otros» (p. 106).

Los poderes otorgados al gobierno durante el Frente nacional señalan que eran mayormente para lograr, implícita y explícitamente, facultades extraordinarias en las que predominaban las transgresiones en materia legal y las arbitrariedades de carácter general.

El mantenimiento de la seguridad como factor preponderante dentro del estado de sitio ocupaba el primer lugar, lo cual era estimado, según el gobierno, como necesario en razón del deteriorado orden público.

Al existir facultades expresas cuando se trata del mantenimiento del orden público se hace necesario -opinan algunos constitucionalistas- fundamental otorgar facultades implícitas, es decir, en un sistema democrático se hacen necesarias no solamente las facultades explícitas sino que hay lugar a dar una interpretación extensiva a la Constitución para lograr superar crisis reales o presuntas. El estado de sitio como parte de las excepcionalidades a la normatividad constitucional en Colombia, según García (2000):

Ha hecho de las fuerzas armadas un actor político insoslayable en la estructura interna de la toma de decisiones estatales. Así, por ejemplo, la prolongación del estado de sitio en el tiempo ha incidido drásticamente en la configuración de la dinámica gubernamental, de tal manera que las tareas sociales y políticas terminan siendo subordinadas a las funciones policivas orientadas al restablecimiento del orden público. (p.21).

Como se observa, la dinámica de implementación del estado de sitio en Colombia se enmarca en un contexto autoritario y militarista latinoamericano, propiciado por la política estadounidense de la Seguridad Nacional que bajo la construcción del imaginario de «enemigo interno", propició una serie de violaciones a los derechos humanos. Como se presenta a continuación, una de las prácticas recurrentes de los gobiernos colombianos durante el Frente Nacional fueron los Consejos Verbales de Guerra, mediante los cuales se extralimitaban las funciones de las fuerzas castrenses en la búsqueda de «chivos expiatorios». 


\section{LOS CONSEJOS VERBALES DE GUERRA COMO PRÁCTICA VIOLATORIA DE LOS DERECHOS HUMANOS}

El consejo verbal de guerra es un procedimiento establecido en el código penal militar colombiano. A raíz de la huelga de las bananeras de 1926 el gobierno nacional en 1928 creyó necesario que aquellos individuos que hubiesen participado en la huelga fuesen juzgados por tribunales militares. Es así que desde 1928 a través del decreto legislativo nro. 2 se establecieron los consejos verbales de guerra para juzgar a civiles. En aquel momento bajo el estado de sitio el gobierno incita y tolera la violencia hacia los trabajadores, vulnera todos los derechos. Echeverri señala que «todas las personas que estuvieran en contra del gobierno son arrestadas y en su mayoría condenadas por un tribunal militar reunido en Ciénaga» (2014, p. 11).

Como se colige, los consejos verbales de guerra van estrechamente ligados a dos aspectos: por un lado al estado de sitio, el cual facilita la arbitrariedad en razón a las facultades extraordinarias del ejecutivo, y por otro lado, al procedimiento maniqueo pero necesario de las prerrogativas otorgadas a los militares, para poder juzgar con severas penas a individuos presuntamente autores de delitos en contra del Estado. Además de lo dicho, estos Consejos Verbales de Guerra fueron mediáticos y muy útiles en cuanto a generar escenarios de zozobra por parte del Estado.

Para mirar el cúmulo de arbitrariedades que traen consigo estos procedimientos, se analizará en particular el consejo de guerra seguido contra cuatro individuos acusados de la muerte del general del Ejército, Ramón Arturo Rincón Quiñones, asesinado en Bogotá el 8 de septiembre de 1975.

El conjunto de la opinión pública nacional prestará atención a este caso porque muestra las irregularidades de fondo en el procedimiento penal de los imputados, sirve como antecedente en el caso de obreros acusados de actos subversivos y se vincula con uno de los escándalos de corrupción en las fuerzas militares más conocidos de finales de la década de los setenta: el caso de los sobornos en las fuerzas militares de la Lockheed Aircaft Corporation ${ }^{4}$. Al respecto, a comienzos de febrero de 1976 se estalló el escándalo mundial por los sobornos de la Lockheed Aircaft Corporation a gobernantes, congresistas y altos mandos militares de países europeos, asiáticos y latinoamericanos (principalmente

4 Consúltese: Alternativa. Nro. 89. Febrero 16 al 23 de 1976. P. 2-7. Alternativa. Nro. 89. Julio 8 al 16 de 1976. P. 4-5. Alternativa. Nro. 80. Julio 16 al 23 de 1976. P. 6. Voz Proletaria. 30 de septiembre al 6 de octubre de 1976. P. 5. Voz Proletaria. 28 de octubre al 3 de noviembre de 1976. P. 1 y 3. 
Italia, Holanda, Japón, Irán y Colombia). Los sobornos los hacía la empresa armamentística con la finalidad de que las fuerzas militares de los respectivos países compraran aviones y material de guerra a esta empresa; en el caso colombiano la investigación se remontaba a 1964 con la compra de tres aviones Hércules. Mientras que en los otros países se presentaron destituciones de cargos e incluso encarcelamientos por estos hechos, en Colombia, aunque el presidente prometió una «investigación exhaustiva», el delito quedó impune. Sumado al descrédito que tenían las fuerzas militares por el soborno, el abogado Miguel Antonio Cano, que llevaba el caso, sostuvo que el asesinato del general Ramón Arturo Rincón Quiñones obedeció a que tenía información sobre estos sobornos.

El proceso llevado a cabo por la Justicia Penal Militar marca un precedente en la medida que fue el primer asesinato contra un general del Ejército orientado por un grupo insurgente y por lo controvertido que fue el fallo en contra de las personas imputadas. En un comunicado enviado a diversos medios informativos, el Ejército de Liberación Nacional (ELN) confirmó la autoría del atentado contra el general Rincón Quiñones. Allí sostiene enfáticamente que ninguna de las personas condenadas en el Consejo de Guerra tuvo «absolutamente nada que ver» con ese hecho y que además ninguno pertenece a esa organización guerrillera. De igual forma, aclara que «jamás será conducta del ELN utilizar personas ajenas, y menos delincuentes comunes, para cumplir sus acciones revolucionarias» (Alternativa. Nro. 67, 1976, p. 13). El ELN publica prácticamente 2 meses después del fallo contra los imputados debido a la presión y el debate que generó en la opinión pública la falta de garantías procesales.

Las pruebas que utilizó el fiscal fueron el testimonio del zapatero Belisario Duarte, testigo presencial de los hechos, y las afirmaciones de Ricardo Quiroga, uno de los inculpados como autor material de la muerte del alto oficial. El zapatero Duarte implicó en su declaración al campesino Carlos Arturo Isaza, al obrero Heriberto Ramírez, al abogado Uriel Casadiegos y a otro sindicado de nombre Ricardo Quiroga, argumentando que el primero era quien manejaba el vehículo donde se movilizaban los presuntos asesinos, cuestión que se desfiguró cuando se comprobó que Isaza no sabía manejar y ni siquiera conocía Bogotá. Posteriormente se pudo comprobar que «Belisario Duarte tenía antecedentes penales y que estaba cooperando con las autoridades militares para sanear sus cuentas con la justicia» (Alternativa. Nro. 67, 1976, p. 3).

Al comprobar que efectivamente Isaza no sabía manejar ni conocía Bogotá, el fiscal, mayor Gerardo Nungo, pidió la absolución del campesino, pero validó lo dicho por Duarte contra los otros tres sindicados, dividiendo el testimonio e incurriendo en una irregularidad al darle credibilidad a un testigo que ya había falseado su versión (El Tiempo. 21 de enero de 1976. P. 2A). 
El segundo testigo utilizado por la Fiscalía fue Ricardo Quiroga, ya que acusado como autor material, había reconocido su culpabilidad e inculpado a Heriberto Ramírez, a Uriel Casadiegos y a otro sospechoso de nombre German Villamil como asesinos del general. Quiroga se retractó de todo lo dicho inicialmente, revelando que había sido coaccionado y dirigido en todos los aspectos de su confesión por un coronel del B-2 (Alternativa. Nro. 67, 1976, p. 3). Sostuvo de igual forma que en el B-2 le dijeron que mantuviera su historia inicial y le ayudarían con un lío judicial que tenía por estafas a la Caja Agraria; también lo instruyeron en decir que solamente había disparado contra el chofer del general Rincón Quiñones, quien quedó herido, y así solamente lo culparían por lesiones personales y saldría pronto de la prisión. En una tercera audiencia Ricardo Quiroga insistió en la veracidad de lo dicho inicialmente, negando categóricamente lo mencionado sobre las ventajas que le brindaba el B-2 y, en efecto, fue condenado por disparar sobre el chofer (El Tiempo. 6 de enero de 1976. P. 1 y 8A); los otros tres sindicados fueron condenados a cien años de prisión.

En cuanto al caso de Germán Villamil, se le sindicó en un inicio como autor material del atentado. El general Fernando Landazábal Reyes, comandante de la Brigada de Inteligencia Militar (BIM), afirmó que tenía pruebas que demostraban que Villamil fue uno de los que disparó contra Rincón Quiñones; sin embargo, cuando se demostró que en el momento del atentado Villamil estaba bajo anestesia en la Clínica Santo Tomás, le cambiaron la sindicación por la de autor intelectual (Alternativa. Nro. 57. 1975. P. 4).

Al abogado Uriel Casadiegos también se le condenó por habérsele encontrado un mapa del sector del crimen del general en su oficina. Se comprobó que el plano correspondía a un accidente de tránsito de uno de sus clientes, el cual fue corroborado por el propio autor del choque (Alternativa. Nro. 67, 1976, p. 4). Los abogados defensores lograron establecer que el «testigo estrella», Ricardo Quiroga, había estado en un hostal en la ciudad de Tuluá el día del asesinato del general.

Por otra parte, el fiscal que llevó el caso, mayor Gerardo Ñungo, durante el proceso hizo una serie de afirmaciones que ponen en evidencia la actuación de la Justicia Penal Militar y que dan por entendido un problema tan serio que es recurrente durante los gobiernos de Alfonso López Michelsen y Julio César Turbay Ayala: la falta de garantías para los sindicados. Para el fiscal de este caso: «quien investiga, debe ser el mismo que falla»; «es preferible condenar a un inocente que absolver a un culpable (...) para evitar las dilaciones de la justicia ordinaria, habría que convertir el indicio en prueba suficiente para condenar a un sindicado» (Alternativa. Nro. 67, 1976, p. 3). 
La aplicación de Consejos Verbales de Guerra fue recurrente en este periodo y en muchas ocasiones fueron empleados contra el movimiento obrero. Cuando pasaban a la justicia ordinaria por levantamiento del estado de sitio, muchos de los fallos cambiaban sustancialmente y se ratificaba la inoperancia de la Justicia Penal Militar. Justamente para el mes de septiembre, a un año del asesinato del general Rincón Quiñones, el fiscal noveno del Tribunal Superior de Bogotá, Enrique Romero Rincón, pidió la revocatoria de las sentencias contra los cuatro imputados por considerar que los testigos fueron manipulados y se violaron las más elementales normas del procedimiento penal. Además de este caso, fue anulado el «Consejo de Guerra del Siglo», contra el ELN, en 1969, y la declaratoria de nulidad del Consejo de Guerra contra el EPL, a finales de 1975, en Medellín (Alternativa. Nro. 98. 1976. P. 2-5).

Como se observa, muchos de estos procedimientos eran inútiles y lo que pretendían era la búsqueda de «chivos expiatorios» que dieran cuenta de la «efectividad» de la justicia en materia penal en la opinión pública colombiana.

Paralelo a la represión del establecimiento en contra de las organizaciones populares y a la búsqueda de «chivos expiatorios» para generar sentimientos de «orden y seguridad» en el imaginario social, las guerrillas realizaban acciones que a todas luces evidenciaban un escenario de amenaza y zozobra para las élites políticas y económicas, los mandos militares e incluso la dirigencia sindical. El ELN fue una de las organizaciones guerrilleras que más desarrolló atentados contra la élite militar y económica durante 1975; se atribuyó a través de un comunicado el asesinato del teniente coronel José Joaquín Romero, el 16 de mayo de 1975 (Alternativa. Nro. 75. 1975. P. 13); de igual forma, el asesinato del gerente de Sofasa en Medellín, Jean Ives Colomb (Alternativa. Nro. 71. 1973. P. 26). No obstante, entre 1976 y 1980, la organización que más amenazó, retuvo, atentó y asesinó a la élite económica fue el M-19; tanto así, que a los pocos días de la condena contra los imputados de la muerte del general Rincón Quiñones, el M-19 decide secuestrar a José Raquel Mercado y hacerle un juicio revolucionario.

\section{EL DELITO POLÍTICO DURANTE EL FRENTE NACIONAL}

Durante el Frente Nacional el delito político prácticamente desapareció y dio paso a lo que en los siguientes años sería la denominación de «terrorista» a todos los que efectivamente eran delincuentes políticos. Montoro (2000) argumenta que en el delito político hay una «acción materialmente lícita (bona per se o éticamente indiferente) que por «razones políticas» es tipificada como delictiva. Dentro de esas acciones materialmente lícitas que el poder convierte en delictivas (mala quia prohibita) cabe distinguir dos tipos: a) De un lado, las 
acciones de signo positivo que, de ordinario, consisten en el ejercicio de determinados derechos fundamentales (libre expresión del pensamiento, libertad de asociación y de reunión, derecho de huelga, etc.) incluido el derecho de resistencia al poder ilegítimo. b) De otro lado, acciones de signo negativo (omisiones)» (Montoro, 2000). En otros términos, dentro de los delitos políticos esta hace mención al trato que debe dársele a aquellos que cometan dichos delitos con la intención de buscar un bien común, a individuos que tengan fines generosos, lo que da cabida para considerar que no solo la rebeldía entra en la categoría de delito político, sino también aquellos que tengan algún fin altruista. En tal sentido, el delito político es esencialmente «una construcción artificial del legislador mediante la cual, y por razones políticas, una conducta per se lícita o éticamente indiferente pasa a ser considerada como ilícita y, por ello, sancionada con una pena» (Montoro, 2000, p. 155).

En los Consejos Verbales de Guerra, se castigaba fundamentalmente delitos políticos. Como en la legislación colombiana se encuentran ciertos vacíos en cuanto a lo que respecta a los delitos políticos, este aspecto era bien utilizado por el gobierno y por supuesto por los tribunales militares. Esto era posible, en la medida que no se tiene una definición concreta acerca de qué es o lo que constituye un delito político, así como tampoco a quiénes se les puede llegar a considerar como delincuentes políticos, ya que esto tiene aspectos completamente subjetivos y va a variar dependiendo de la persona que se encuentre en el poder.

Si bien los Consejos Verbales de Guerra eran «efectivos» cuando de juzgar civiles se trataba en términos de miedo o terror hacia esos tribunales e incluso hacia el mismo gobierno, esto no ocurría cuando era personal adscrito a la fuerza pública el sometido a tal jurisdicción. En ellos se aplicaba el fuero penal militar, de tal modo que por ningún delito fuesen a ser juzgados por jueces civiles; buscaban el perdón judicial en casos de delitos graves, lo cual conducía a un alto grado de impunidad. Al contrario, los militares no consideraban que los civiles dentro de su jurisdicción penal pudieran ser objeto de amnistías o indultos. Sin embargo, estas leyes de amnistía como forma de perdón a nivel penal se encontraban establecidas en el artículo 76, numeral 21; allí se señalaba como facultades del Congreso de la República «Conceder, por mayoría de dos tercios de los votos en cada Cámara, y por graves motivos de conveniencia pública, amnistías o indultos generales por delitos políticos. En el caso de que los favorecidos queden eximidos de la responsabilidad civil respecto de particulares, el Gobierno estará obligado a las indemnizaciones a que hubiere lugar».

La amnistía y el indulto son exclusivamente para los delitos políticos -aunque se incluyan los delitos conexos para facilitarlos y posibilitarlos-, como lo instaura 
la Constitución colombiana. Así, a los acusados en juicios verbales de guerra del cometimiento de un delito tipificado como político actualmente, frente a sus acciones, les fue negado el derecho a la amnistía o al indulto. Cabría resaltar que tipificar todos los delitos que actualmente se consideran como políticos será objeto de otro trabajo.

\section{CONCLUSIONES}

Comprendiendo la situación que viviera la sociedad colombiana en el periodo histórico de 1958-1974, es considerable cómo la justicia se produjo con parcialidad, como en el caso de los tribunales militares de justicia verbal, en los que se abusó de la posición de autoridad y se llevó a la sociedad hacia las prácticas del horror, del sinsentido de la aplicación de un orden jurídico no social. Del mismo modo, se deslegitimó la práctica política y el ordenamiento jurídico y se llevó a la sociedad a generar un rechazo a la forma de gobierno, que encabezada por la corrupción anunciada en la traición a la promesa instaurada en la campaña política, dio al traste en las instancias del poder, con la común unidad celebrada en las elecciones.

El gobierno nacional, materializado en la figura de poder de López Michelsen, desestabilizó la vida nacional, en contravía de la justicia, aplicando un ordenamiento jurídico injusto e ilegítimo que permite observar las prácticas políticas de una élite enferma de poder, que solo necesita del pueblo como empresa electoral y no mide sus actuaciones en el interior del Estado.

En definitiva, la práctica del consenso traslapado cesó en gobiernos caducos por su corrupción e inoperancia social, que abatieron el valioso proceso social del reconocimiento de los derechos de los trabajadores y trajeron de por sí el descontento e inestabilidad, que son el pan de cada día de la ciudadanía colombiana, que aún reclama en medio del conflicto, por un Estado responsable de los intereses del colectivo, y que se encause hacia la verdadera justicia.

Las medidas represivas como los «Consejos Verbales de Guerra» son procesos dirigidos por la justicia penal militar contra civiles, en los cuales se juzga y condena sin tener en cuenta elementales garantías procesales y cuyas confesiones, en muchas ocasiones, son extraídas por medio de torturas. Los procedimientos encauzados para buscar la verdad de los acontecimientos y la responsabilidad de actores materiales e intelectuales traerán consigo un elevado manto de impunidad que la sociedad en su conjunto empieza a naturalizar. En esencia, los Consejos Verbales de Guerra son las instancias objeto de estudio a través del prisma que integra la filosofía política rawlsiana, llegando a un trasfondo social, político e histórico, del que se deducen las 
formas de interpretar en derecho la violación sistemática de los derechos humanos.

Se planteó, a lo largo del artículo, la necesidad de estrategias que impidan la repetición de este tipo de eventos, que eliminan el consenso como base de la legitimidad del accionar del Estado frente a la sociedad, más cuando la común-unidad ha elegido a sus representantes para que cumplan con un programa político y no se pierdan en demagogias de baja categoría, solo por escalar las altas esferas del poder en traición de la multitud, ávida de justicia para una sociedad en gestación política.

Por otra parte, quedó claro que el triunfo democrático se observa en la historia del pensamiento, enfocándonos en la concientización que genera el movimiento hacia la educación de todos para la construcción del proyecto político. Desde Aristóteles hasta Rousseau, de la ética a Nicómaco al Emilio, desde la creencia en la república francesa hasta el testimonio de Tocqueville sobre la democracia que se basa en la identidad de costumbres, sentimientos y creencias, todos concuerdan con la imperiosa necesidad de sumar educación a la política, para la realización de un sistema político ético. Como lo comprende Barragán (2012):

En Colombia se requiere el establecimiento de una mentalidad democrática para erradicar el mal congénito de la violencia, desterrar los demonios de la corrupción, establecer pautas para el diálogo, capaces de construir el consenso legítimo que lleve al mandar obedeciendo que plantea la política de la liberación de Dussel; hacia la interiorización de los principios democráticos que como valores se expresan en la solidaridad, el respeto en todas sus formas (al otro, al bienestar común, a los bienes colectivos) y en la provisión de las condiciones suficientes para que la dignidad humana se realice y se mantenga en la institucionalidad que fundamenta la Constitución de 1991, como marco normativo y de referencia para la estructura de la sociedad (p. 116).

Las sociedades bien ordenadas tienen el deber de defender a los pueblos y a los derechos humanos. Pero fundamentalmente, «las sociedades bien ordenadas tienen el deber, de raigambre kantiana, de conseguir que todas las sociedades se adhieran al derecho de gentes y se conviertan en miembros de la sociedad de los pueblos» (Rawls, 1998: 182).

Para llegar al «ethos» democrático, la educación se presenta como la posibilidad de dar luz al sendero. En la plena interiorización de los valores, considerando a la democracia como un proyecto ético de la construcción de la sociedad que finalmente se escinde de cualquier control foráneo, que empieza a pensar por sí misma, con sujetos incluyentes e ilustrados, que den pasos de gigante en las azarosas tierras en proceso globalizante hegemónico. 
Henry Torres Vásquez, Eder Maylor Caicedo Fraide,

Daniel Alfonso Barragán Ronderos

\section{REFERENCIAS}

Alternativa. Nro. 57.1 al 7 de diciembre de 1975, p. 4.

Alternativa. Nro. 71. Del 23 de febrero al 1 de marzo de 1973.

Alternativa. Nro. 67. Enero 26 a febrero 2 de 1976, p. 3.

Alternativa. Nro. 98. Septiembre 13 al 20 de 1976, p. $2-5$.

Alternativa. Nro. 75. Del 22 al 29 de marzo de 1975, p. 13.

Alternativa. Nro. 65. Diciembre 26 de 1975-enero 6 de 1976, p. 22.

Aristóteles. (1991). La Política. México: Ed. Porrúa Hermanos.

Barker, J. (2004). El sinsentido del terrorismo. Barcelona: Edit. Fundación Intermón.

Barragán Ronderos, D. A. (2011). La Globalización, un proceso hegemónico mundial: orígenes, repercusiones y actualidad. Revista Via Iuris, núm. 10, enero-junio, 2011, pp. 103-111. Fundación Universitaria Los Libertadores. Bogotá, Colombia.

Barragán Ronderos, D. A. (2012). La política de la liberación. Revista Via Iuris, núm. 12, enero-junio, 2012, pp. 105-117. Fundación Universitaria Los Libertadores. Bogotá, Colombia.

Barry, B. (1995). Teorías de la justicia. Barcelona: Gedisa.

Beitz, C. (1979). Political Theory and International Relations. Princeton: Princeton University Press.

Borja Jiménez, E. (2013). Irretroactividad y retroactividad para los peligrosos o socialmente indeseables. Cuadernos de Derecho Penal. Derecho Penal y Derecho Internacional. Universidad Sergio Arboleda.

Botero, J. J. (2004). Con Rawls y contra Rawls. Universidad Nacional de Colombia. Bogotá: Editorial UN.

Chaparro, C. (2013). Amnistía e indulto en Colombia 1965-2012. Universidad Nacional de Colombia. Bogotá.

CIDH (2006). Caso Almonacid Arellano y otros contra Chile, Sentencia de 26 de septiembre de 2006, argumento núm. 151. 
Contreras Peláez, F. J. (2009). Revista Internacional de Pensamiento Político. I Época. Vol. 4. [137-142]. ISSN 1885-589X

De Roux, C. V. (2000). Humanización del conflicto y proceso de paz, en Camacho Guizado, Álvaro y Leal Buitrago, Francisco, compiladores, Armar la paz es desarmar la guerra, Centro de Estudios de la Realidad Colombiana (CEREC), Bogotá.

Echeverri Duque, S. (2014). Los estados de excepción en Colombia: un estudio de caso, Revista Ces, Derecho, vol. 5 núm. 1, Medellín, Colombia, enero-junio 2014, p. 11.

El Tiempo. (6 de enero de 1976), pp. 1 y 8 A.

García Villegas, M. (2000). Estado, derecho y crisis en Colombia, Revista Estudios Políticos, núm. 17, Medellín, p. 21.

Hobbes, T. (1979). Leviatán. Madrid: Editorial Nacional.

Iturralde, M. (2003). Guerra y derecho en Colombia: el decisionismo político y los estados de excepción como respuesta a la crisis de la democracia, Revista de Estudios Sociales, Universidad de los Andes, Colombia.

Kant, I. (1998). Sobre la paz perpetua. Madrid: Alianza.

Kant, I. (2001). El derecho de gentes y «Una revisión de las idea de razón pública». Barcelona: Paidós.

Kant, I. (2002). Teoría de la justicia. México: F. C.E.

Kant, I. (2005). La metafísica de las costumbres (4 ed.). Madrid: Tecnos.

Lipset, S. (1992). Diez textos básicos de Ciencia política. Barcelona: Editorial Ariel S. A.

Martínez, R. A. (2007). La investigación en la práctica educativa. Madrid: CIDE.

Montoro, B. A. (2000). En torno a la idea de delito político (Notas para una ontología de los actos contrarios a Derecho), http:/ / revistas.um.es/analesderecho/article/ view/57601/55481, [Consulta 21 de enero de 2016].

Nagel, T. (2003). Rawls y el Liberalismo. The Cambridge Companion to Rawls. Cambridge University Press.

Pérez Zafrilla, P. J. (2009). El derecho de gentes desde la perspectiva de John Rawls. Criterio Jurídico, núm. 9-1, julio, pp. 106-126. Id. vLex: VLEX-67139952 - http: //vlex.com/vid/67139952 
Rachels, J. (1999). The Elements of Moral Philosopphy. McGraw-Hill.

Rawls, J. (1971). The Theory of Justice. Harvard University Press, Cambridge Mass.

Rawls, J. (1971). Teoría de la justicia. México: Fondo de Cultura Económica.

Rawls, J. (1993). Political Liberalism. Nueva York: Columbia University Press.

Rawls, J. (1996). El liberalismo político. Bogotá: Fondo de Cultura Económica.

Rawls, J. (1998) «El derecho de gentes». En: De los derechos humanos. Ed. S. Shute y S. Hurley. Madrid: Trotta.

Rousseau, J. J. (1762). Du contrat social ou Principes du droit politique. Amsterdam.

Rousseau, J. J. (1988). El contrato social o Principios de derecho político. Estudio preliminar y traducción de María José Villaverde. Madrid: Editorial Tecnos.

Scanlon, T. M. (1982). «Contractualism and Utilitarianism» en Utilitarianism and Beyond, A. Sen y B. Williams (eds.), Cambridge University Press.

Tirado Mejía, A. (1978). Colombia: Siglo y medio de bipartidismo, en: Arrubla Mario y otros, Colombia, Hoy. Bogotá: Siglo Veintiuno Editores.

Torres Vásquez, H. (2010). Terrorismo de Estado y Derecho Penal. Bogotá: Editorial Gustavo Ibáñez.

(2009). América Latina: entre el militarismo, la violencia politica y la violencia institucional. El sofisma de la seguridad. Valencia, España: Editores Valencianos.

Wolff, R. P. (1977). Understanding Rawls. Princeton University Press. (Traducción de Marcial Suárez. 1981. Para comprender a Rawls. F. C. E. México). 